\title{
O estresse em acadêmicos de enfermagem no ensino clínico: uma pesquisa integrativa
}

\author{
Stress in clinical education-related nursing academics: an integrative research \\ Estrés en la academia de enfermería clínica relacionada con la educación: una \\ investigación integrativa
}

Flávia dos Santos Lugão de Souza ${ }^{1 *}$, Renata da Silva Hanzelmann ${ }^{1}$, Joanir Pereira Passos ${ }^{1}$

\section{RESUMO}

Objetivo: Identificar os fatores predisponentes ao estresse em acadêmicos de enfermagem em ensino clínico registrados na literatura científica. Métodos: Consta de uma Revisão Integrativa da literatura. Os dados foram coletados a partir de publicações nas bases de dados Scientific Electronic Library Online (SCIELO) e Biblioteca Virtual em Saúde (BVS). Foram selecionados 212 artigos, após os critérios de inclusão somente 18 artigos foram utilizados no estudo. Resultados: $O$ ensino clínico é entendido como um instrumento para formação dos profissionais de enfermagem, nele se desenvolvem habilidades profissionais e se aperfeiçoam técnicas e procedimentos realizados diariamente no exercício da profissão. Os estudantes de enfermagem experimentam elevados níveis de estresse, pela maior proximidade e permanência junto a pacientes. Os fatores predisponentes ao estresse dependem de cada indivíduo, englobando fatores assentados na personalidade, autoestima e resistência orgânica. Os acadêmicos de enfermagem estão cercados diariamente por conflitos, devido estar em contato com a dor e o sofrimento humano. Considerações finais: Verificou-se que o estresse está presente em todos os períodos de formação acadêmica, com tendência a se intensificar com o início das atividades práticas de ensino clínico. Quanto mais avançado o período em que o aluno se encontra, maior seu nível de estresse.

Palavras-chave: Estresse ocupacional, Estágio clínico, Enfermagem, Estudantes.

\section{ABSTRACT}

Objective: To identify the factors predisposing to stress in nursing students in clinical teaching registered in the scientific literature. Methods: It consists of an Integrative Literature Review. Data were collected from publications in the Scientific Electronic Library Online (SCIELO) and Virtual Health Library (BVS) databases. 212 articles were selected, after applying the filters, only 18 articles were used in the study. Results: Clinical teaching is understood as an instrument for training nursing professionals, where professional skills are developed, and techniques and procedures performed daily in the exercise of the profession are perfected. Nursing students experience high levels of stress, due to the greater proximity and permanence with patients. The factors predisposing to stress depend on everyone, encompassing factors based on personality, self-esteem and organic resistance. Nursing students are surrounded daily by conflicts, due to being in contact with human pain and suffering. Final considerations: It was found that stress is present in all periods of academic training, with a tendency to intensify with the beginning of practical clinical teaching activities. The more advanced the period the student is in, the greater his level of stress.

Keywords: Occupational stress, Clinical internship, Nursing, Students.

\section{RESUMEN}

Objetivo: Identificar los factores que predisponen al estrés en estudiantes de enfermería en la enseñanza clínica registrada en la literatura científica. Métodos: Consiste en una Revisión Integral de Literatura. Los datos se recopilaron de publicaciones en las bases de datos de la Biblioteca Electrónica Electrónica (SCIELO) en línea y de la Biblioteca Virtual de Salud (BVS). Se seleccionaron 212 artículos, después de aplicar los filtros, solo se utilizaron 18 artículos en el estudio. Resultados: La enseñanza clínica se entiende como un instrumento para la formación de profesionales de enfermería, donde se desarrollan habilidades profesionales y se perfeccionan técnicas y procedimientos que se realizan diariamente en el ejercicio de la profesión. Los estudiantes de enfermería experimentan altos niveles de estrés, debido a la mayor proximidad y permanencia con los pacientes. Los factores que predisponen al estrés dependen de cada individuo, abarcando factores basados en la personalidad, la autoestima y la resistencia orgánica. Los estudiantes de enfermería están rodeados diariamente de conflictos, debido al contacto con el dolor y el sufrimiento humanos. Consideraciones finales: Se encontró que el estrés está presente en todos los períodos de formación académica, con una tendencia a intensificarse con el inicio de las actividades prácticas de enseñanza clínica. Cuanto más avanzado es el período en que se encuentra el estudiante, mayor es su nivel de estrés.

Palabras clave: Estrés ocupacional, Práctica clínica, Enfermería, Estudiantes.

1 Universidade Federal do Estado do Rio de Janeiro (UNIRIO), Rio de Janeiro - RJ.

*E-mail: flavia.l.s@terra.com.br

SUBMETIDO EM: 6/2020

ACEITO EM: 7/2020

PUBLICADO EM: 9/2020 


\section{INTRODUÇÃO}

Vários autores têm discutido o tema estresse e divulgando seus resultados para explicar esse evento que afligem a vida humana. $O$ estresse é definido como qualquer estímulo que demande do ambiente externo ou interno e que exceda as fontes de adaptação de um indivíduo ou sistema social (BUBLITZ S, et al., 2012).

A prática do estágio na área da saúde, combinado com à formação do aluno, apesar da importância assumida pelos próprios acadêmicos e pelas instituições formadoras, também pode ser fonte de sofrimento e conflitos.

Vale ressaltar que, o aluno pode evoluir com um grau de esgotamento psicológico e físico devido a quantidade de atividades a realizar, noites mal dormidas para dar conta dos estudos, atividades e trabalhos acadêmicos, após jornada diária de estágio. Além disso, é sugerido ao acadêmico que participe de outras atividades tais como: grupos de pesquisa, projetos de extensão, monitoria, eventos, além da realização de cursos de atualização (PEREIRA CA, et al., 2010).

Neste contexto, o Estágio Curricular Supervisionado (ECS) traz importante contribuição para a formação do futuro profissional de Enfermagem, tendo em vista tratar-se de uma atividade acadêmica fundamental para este processo de formação. O ECS propicia que o estudante entre em contato direto com a realidade de saúde da população, o que pode ser considerado de grande importância para o seu desenvolvimento pessoal e profissional, bem como para a consolidação dos conhecimentos adquiridos no decorrer do curso de graduação, por meio da relação teoria-prática (LIMA TC, et al., 2014).

Para Torquato JA, et al. (2010) o estresse é uma experiência de tensão, irritação onde o organismo reage a componentes físicos ou psicológicos quando há uma situação que provoque medo, excitação ou confusão. Os autores Oliveira LA, et al. (2014) relatam que os fatores essenciais para o desenvolvimento do estresse que se situam na dimensão interativa homem/meio/adaptação, estes podem ocasionar crescimento e desgastes, além de ser intrínseca a condição de viver. O ser humano deve aprender a coexistir com o ritmo de vida agitado, desgastante, com carência de tempo para o lazer e descanso, alimentação inadequada e cobrança excessiva.

A enfermagem é uma dessas profissões estressantes e, desde a formação acadêmica, o aluno se depara com situações que exigem tomadas de decisões importantes no cuidado do paciente; a insegurança e a ansiedade, decorrentes desse processo, podem desencadear ou piorar o estresse. Características como alto nível de habilidades cognitivas, disposição e atitudes proativas são constantemente requeridas tanto dos enfermeiros que atuam na prática como dos estudantes de enfermagem. Dessa forma, o estresse e a depressão podem prejudicar o desempenho tanto do acadêmico quanto do enfermeiro (MURAKAMI K, et al., 2019).

Tendo em vista disso, a descrição de estudos que abordem o tema estresse em estudantes de enfermagem é de rica necessidade, evidenciando áreas de maior concentração da patologia e facilitando a organização de estratégias e ações de medidas preventivas para a redução do estresse, diminuindo suas consequências para a saúde do enfermeiro em suas atividades laborais. Os resultados esperados poderão despertar reflexões que gerem intervenções no âmbito assistencial de enfermagem e, possivelmente, em outras áreas de conhecimento sobre o estresse relacionado ao aluno em campo de estágio.

Para o desenvolvimento do estudo foi escolhido o tema "O estresse em acadêmicos de enfermagem no ensino clínico" trazendo os seguintes questionamentos como questões norteadoras: O estágio clínico é fator preponderante do estresse em alunos de graduação em enfermagem? Quais os principais sintomas apresentados pelo aluno diante do estresse relacionado ao estágio clínico? O presente estudo tem como objetivo identificar os fatores predisponentes ao estresse em acadêmicos de enfermagem em ensino clínico registrados na literatura científica.

\section{MÉTODOS}

O presente estudo caracteriza-se como uma revisão integrativa da literatura. A pesquisa teve seu início com o levantamento do acervo referente ao tema estudado a partir de publicações nas bases de dados SCIELO e na BVS. Os descritores foram selecionados na base de dados Descritores em Ciências da Saúde 
(DECS), sendo eles: "estresse ocupacional"; "estágio clínico"; "enfermagem"; "estudantes". Esses descritores conectados pelo operador booleano AND, nortearam a busca pelos estudos científicos, orientados pela seguinte pergunta de pesquisa: "O estágio clínico é fator preponderante do estresse em alunos de graduação em enfermagem?

O corte temporal do estudo foi caracterizado nos anos de 2010 a 2019. Para a seleção dos artigos foram feitas pesquisas nas bases de dados eletrônicas SCIELO e BVS. Nessa etapa, a escolha dos artigos utilizados se deu a partir da afinidade com o tema escolhido. A coleta de dados e a análise dos resultados foram feitas nos meses de maio a setembro de 2019.

Para inclusão dos artigos foram estabelecidos os seguintes critérios: Texto completo disponível, Idioma português e inglês, corte temporal 2010 a 2019 e publicações direcionadas para o tema "Estresse em acadêmicos de enfermagem relacionado ao ensino clínico". Os critérios de exclusão adotados foram: estudos em outro idioma, além do português e inglês, trabalhos não disponíveis na íntegra, artigos não liberados gratuitamente e que não atendessem o objetivo de estudo.

Após busca realizada nas bases de dados obtivemos 212 artigos, a partir dos critérios de inclusão e exclusão somente 18 artigos foram aproveitados para a elaboração do estudo. Os dados foram coletados, resumidos e organizados a fim de que pudéssemos atingir o objetivo proposto em questão. Quanto aos descritores pesquisados, identificamos na base de dados SCIELO 11 artigos $(61,1 \%)$ e na base de dados BVS 7 artigos (38,8\%). Foram confeccionados 2 fluxogramas para melhor visualização dos dados relatados acima. Abaixo é apresentado os dados da seleção dos artigos após a aplicação dos filtros na base SCIELO (Figura 1).

Figura 1 - Seleção dos artigos após aplicação dos filtros na base SCIELO.

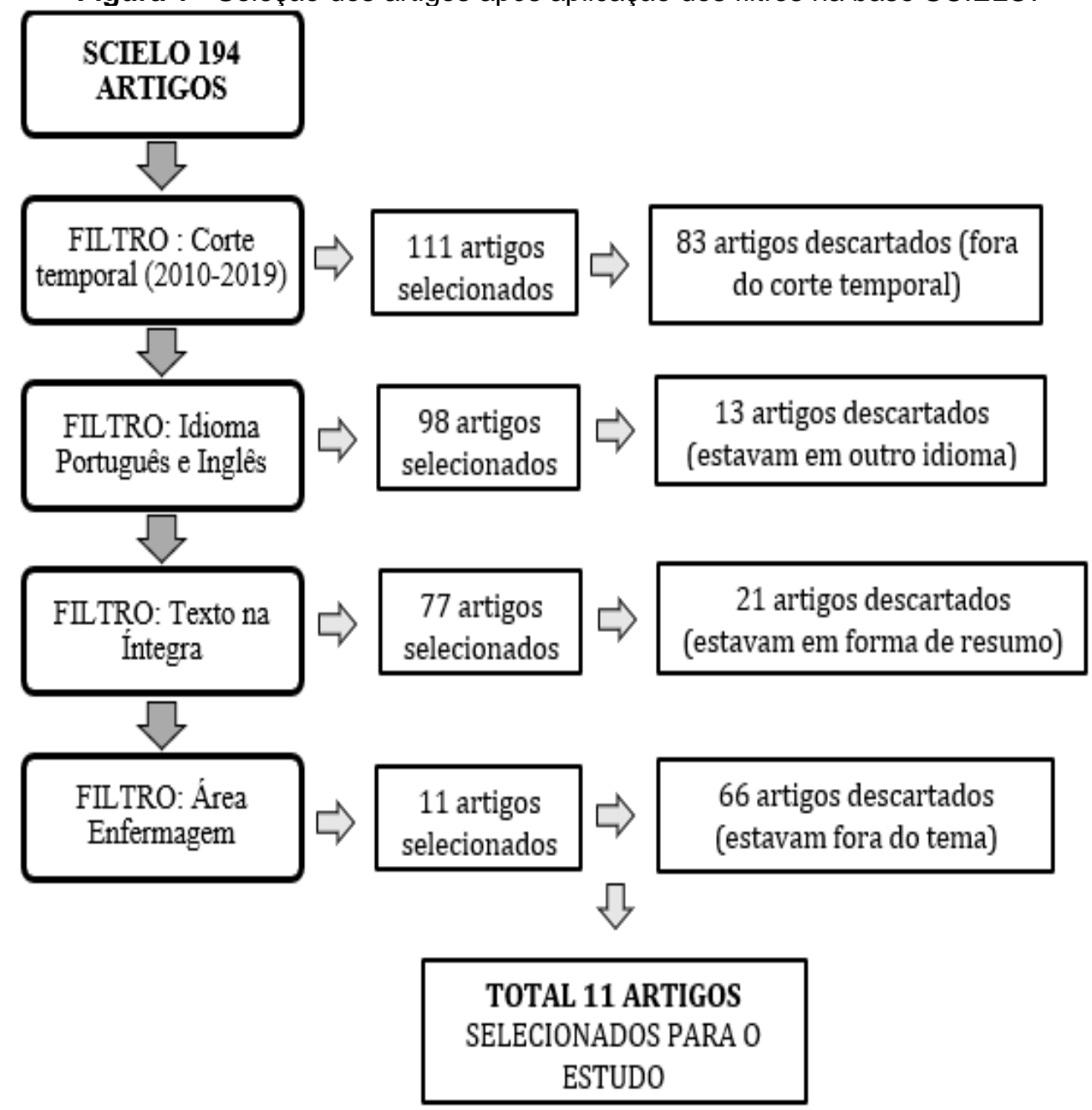

Fonte: Souza FSL, et al., 2019. 
Abaixo é apresentado os dados da seleção dos artigos após a aplicação dos filtros na base BVS (Figura 2).

Figura 2 - Seleção dos artigos após aplicação dos filtros na base BVS.

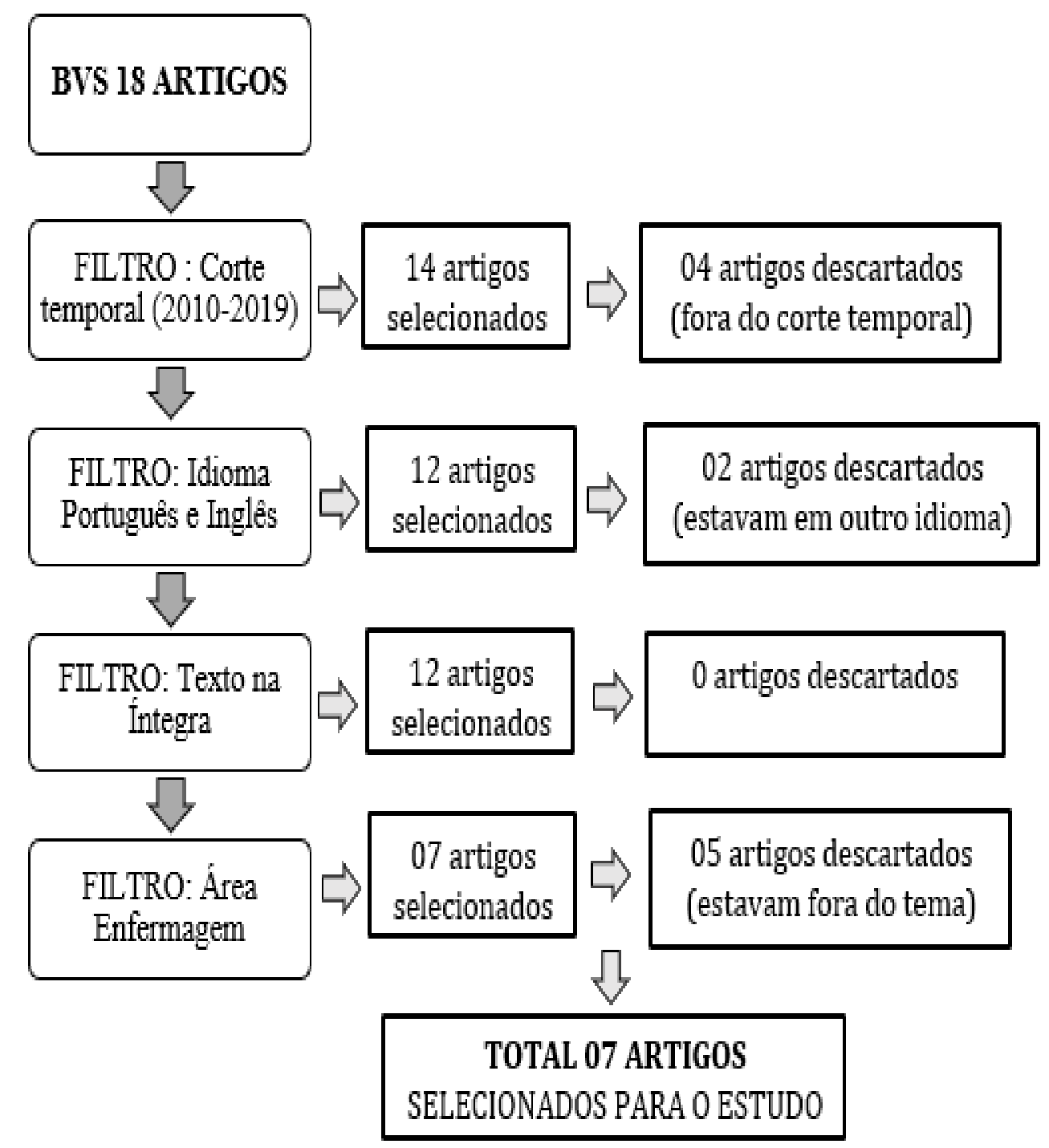

Fonte: Souza FSL, et al., 2019.

\section{RESULTADOS}

Para a realização da análise e discussão dos dados identificados após a leitura dos artigos, levou-se em consideração o título, os autores, o ano de publicação, a fonte, a metodologia dos estudos. A partir dessas variáveis, foi possível organizar os artigos selecionados nas bases com os critérios de inclusão e exclusão adotados nesse estudo (Quadro 1).

Dos 18 artigos selecionados para a elaboração do estudo, observamos que 2 artigos foram extraídos da Revista Acta Paulista de Enfermagem (11,11\%). Os outros 16 artigos foram escolhidos em diferentes revistas: Revista Científica Internacional, Revista Brasileira de Enfermagem, Revista de Enfermagem da UFSM, Revista Repositório Científico de acesso aberto de Portugal, Revista Mineira de Enfermagem, Revista de Psicopedagogia, Arquivo Ciência e Saúde, Revista Saúde e Desenvolvimento Humano, Revista de enfermagem da USP, Revista Científica Sena Aires, Revista de Enfermagem, Revista Médica de São Paulo, Revista Texto e Contexto de Enfermagem, Revista de enfermagem do Centro Oeste Mineiro, Revista de enfermagem da UERJ e Brazilian Journal of Health Review, sendo uma publicação em cada revista (5,55\%). 


\section{Revista Eletrônica Acervo Saúde / Electronic Journal Collection Health ISSN 2178-2091}

Quadro 1 - Seleção dos artigos com base nos critérios de inclusão e exclusão.

\begin{tabular}{|c|c|c|c|}
\hline Título & Autores & Base & Tipo de pesquisa \\
\hline Avaliação do estresse em estudantes universitários. & TORQUATO JA, et al., (2010) & SCIELO & Estudo de coorte transversal. \\
\hline Estágio curricular supervisionado: análise da experiencia discente. & LIMA TC, et al., (2014) & SCIELO & Estudo qualitativo, e documental. \\
\hline Estresse em estudantes de enfermagem: uma revisão integrativa. & BUBLITZ S, et al., (2012) & SCIELO & Revisão integrativa da literatura. \\
\hline $\begin{array}{l}\text { Estressores entre acadêmicos de enfermagem de uma universidade } \\
\text { pública. }\end{array}$ & BUBLITZ S, et al., (2012) & SCIELO & Estudo descritivo, transversal, com abordagem quantitativa. \\
\hline $\begin{array}{l}\text { Percepção de estresse e qualidade de vida dos acadêmicos de } \\
\text { enfermagem no Brasil. }\end{array}$ & BARBOSA T, et al., (2016) & SCIELO & Pesquisa transversal e quantitativa. \\
\hline $\begin{array}{l}\text { O estresse e seus fatores determinantes na concepção dos graduados de } \\
\text { enfermagem. }\end{array}$ & PEREIRA CA, et al., (2010) & SCIELO & Pesquisa descritiva com abordagem quantiqualitativa. \\
\hline $\begin{array}{l}\text { Respostas fisiológicas e emocionais ao estresse em estudantes de } \\
\text { enfermagem: revisão integrativa da literatura científica }\end{array}$ & BENAVENTE SBT e COSTA ALS, (2011) & SCIELO & $\begin{array}{l}\text { Revisão integrativa, de caráter descritivo com abordagem } \\
\text { quantitativa. }\end{array}$ \\
\hline $\begin{array}{l}\text { Expectativas de alunos de enfermagem frente ao primeiro estágio em } \\
\text { instituições de saúde. }\end{array}$ & DIAS EP, et al., (2014) & SCIELO & Estudo de abordagem qualitativa. \\
\hline $\begin{array}{l}\text { Estresse nos acadêmicos de enfermagem de uma universidade } \\
\text { Pública. }\end{array}$ & OLIVEIRA LA, et al., (2014) & SCIELO & Estudo descritivo, com abordagem quantitativa. \\
\hline $\begin{array}{l}\text { Estresse em estudantes de enfermagem: estudo sobre vulnerabilidades } \\
\text { sociodemográficas e acadêmicas. }\end{array}$ & CESTARI VRF, et al., (2017) & SCIELO & Estudo analítico. \\
\hline $\begin{array}{l}\text { Estresse, conceitos, manifestações e avaliação em saúde: revisão de } \\
\text { literatura. }\end{array}$ & NODARI NL, et al., (2014) & SCIELO & Revisão Integrativa da Literatura. \\
\hline $\begin{array}{l}\text { Comparação do estresse em universitários de enfermagem ingressantes e } \\
\text { concluintes do curso. }\end{array}$ & MUSSI FC, et al., (2019) & BVS & Estudo transversal. \\
\hline $\begin{array}{l}\text { Estresse em universitárias (os) de enfermagem e a necessidade do seu } \\
\text { enfrentamento. }\end{array}$ & RIBEIRO FMSS, et al., (2019) & BVS & Revisão Integrativa da Literatura. \\
\hline Percepção de estresse nos acadêmicos de enfermagem. & PRETO VA, et al., (2018) & BVS & Estudo quantitativo e transversal. \\
\hline $\begin{array}{l}\text { Estresse psicológico em estudantes de cursos de graduação da área da } \\
\text { saúde: subsídios para promoção de saúde mental. }\end{array}$ & MURAKAMI K, et al., (2019) & BVS & Estudo do tipo corte transversal. \\
\hline $\begin{array}{l}\text { Fatores percebidos pelos acadêmicos de enfermagem como } \\
\text { desencadeadores do estresse no ambiente formativo. }\end{array}$ & HIRSCH CD, et al., (2018) & BVS & $\begin{array}{l}\text { Estudo quantitativo de caráter descritivo-exploratório e corte } \\
\text { transversal. }\end{array}$ \\
\hline $\begin{array}{l}\text { Estresse no cotidiano de graduandos de enfermagem de um instituto } \\
\text { federal de ensino. }\end{array}$ & SANTANA LL, et al., (2018) & BVS & Estudo quantitativo, transversal e descritivo. \\
\hline $\begin{array}{l}\text { Estresse em acadêmicos de enfermagem: importância de identificar o } \\
\text { agente estressor. }\end{array}$ & SANTOS RJLL, et al., (2019) & BVS & Revisão Integrativa da Literatura. \\
\hline
\end{tabular}

Fonte: Souza FSL, et al., 2019. 
Relacionando a qualificação dos periódicos, sete artigos (38,88\%), apresentaram qualis B2, enquanto cinco artigos (27,77\%) qualis A2, três artigos (16,66\%) qualis B3, dois artigos $(11,11 \%)$ qualis $\mathrm{B} 1$ e um artigo (5,55\%) qualis A3 (Gráfico 1).

Gráfico 1 - Distribuição dos periódicos relacionados ao Qualis.

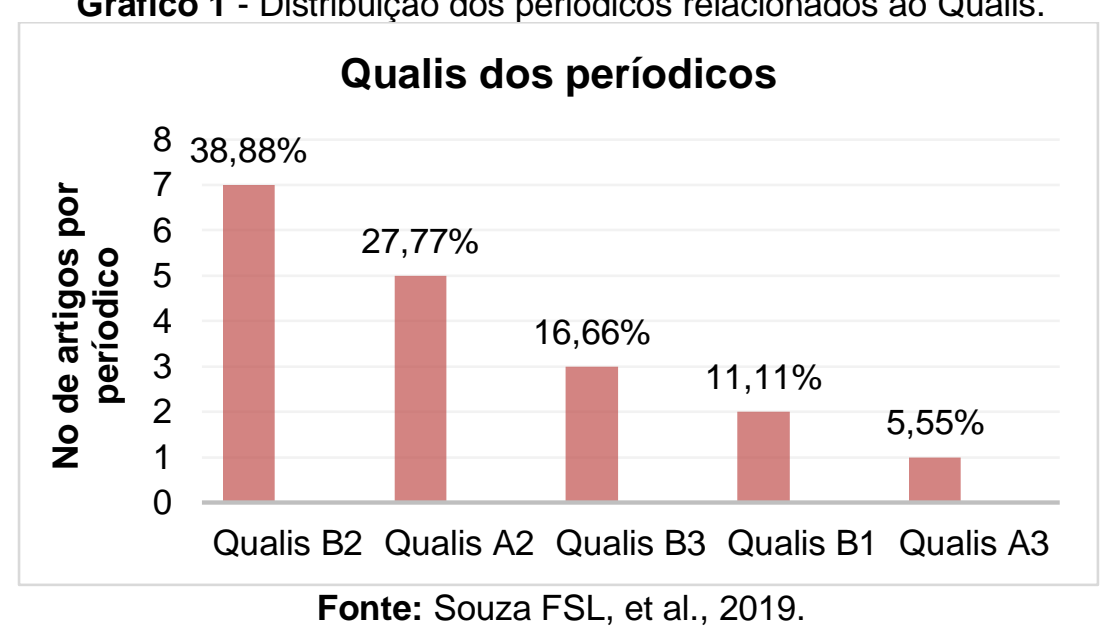

Quanto ao tipo de pesquisa oito artigos (44,44\%) eram pesquisas coorte transversal, quatro artigos $(22,22 \%)$ revisões integrativas, três artigos $(16,66 \%)$ pesquisa qualitativa, um artigo exploratório, uma descritiva quantiqualitativa e um estudo analítico (5,55\% cada) (Gráfico 2).

Gráfico 2 - Distribuição dos artigos quanto ao tipo de pesquisa.

\section{Tipo de pesquisa dos artigos}

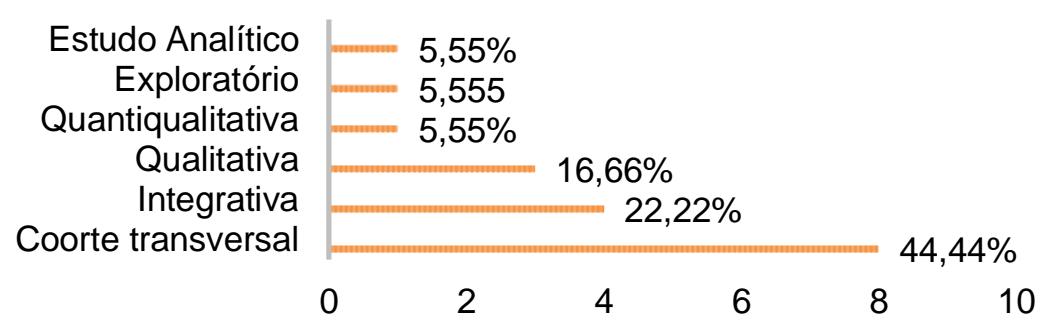

Fonte: Souza FSL, et al., 2019.

Quanto ao ano de publicação dos 18 artigos selecionados, oito artigos têm como anos de publicação 2019 e 2014 divididos igualmente nesses anos, três artigos foram publicados em 2018, dois artigos em 2010 e dois em 2012 e um artigo em cada ano de 2011, 2016 e 2017 (Gráfico 3).

Gráfico 3 - Distribuição dos estudos por ano de publicação.

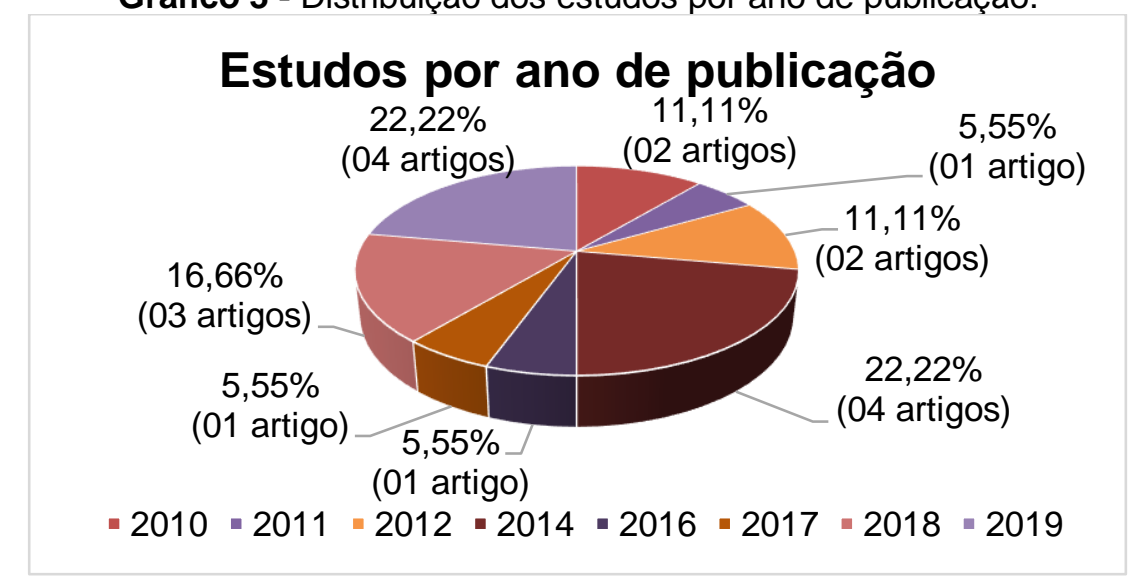

Fonte: Souza FSL, et al., 2019. 


\section{DISCUSSÃO}

\section{Fisiologia do estresse}

O mundo evoluiu e através dessas modificações o ser humano transforma seu modo de agir e interagir com seu ambiente, e em situações e ambientes distantes. As transformações na área tecnológica trouxeram benefícios para o desenvolvimento do homem em seu contexto social, cultural e biológico, porém veio acompanhada de numerosos problemas, expondo-o à fragilidade física e emocional. Nesse contexto surge, o estresse denominado como um dos grandes distúrbios do mundo moderno, e considerado "uma epidemia global" pela Organização Mundial da Saúde (OMS) (LIMA TC, et al., 2014).

Segundo Pereira CA, et al. (2010) o estresse está dividido em dois tipos: eustress - definido como ansiedade desenvolvida nos ambientes em um período de tempo e nas práticas diárias da vida e é produto da ação individual; e distress - o indivíduo evolui com uma quebra no autocontrole biopsicossocial pelo aumento ou ausência de empenho incompatíveis com os ambientes, período de tempo e ação individual.

Segundo Gazzaniga MS, Heatherton TF (2005); Faro A, Pereira M (2013) apud Nodari NL, et al. (2014) o estresse pode ser dividido em 3 fases: estágio de alarme, de resistência e de exaustão. Na fase de alarme, o organismo experimenta um tipo de exaltação, injúria ou fuga do estressor, compreendida como adaptação. A pessoa que se enquadra nessa etapa pode evoluir com vários sintomas, tais como taquicardia, tensão crônica, dor de cabeça, sensação de esgotamento, pressão no peito, extremidades frias, dentre outros.

A fase de resistência, é definida como mobilização das forças defensivas estimulando o córtex adrenal a secretar hormônios adrenocorticóides para a manutenção orgânica, podendo evoluir com ansiedade, medo, isolamento social, oscilação do apetite, impotência sexual e outros. Na fase de exaustão o sistema orgânico está consumido pelo excesso de atividade e pelo alto consumo de energia, podendo evoluir com falência do órgão-alvo, manifestado sob a forma de doenças orgânicas.

Oliveira LA, et al. (2014), descrevem uma quarta fase de evolução do estresse, que se desenvolve entre a fase de resistência e a de exaustão, denominada de quase-exaustão. Nela observa-se a diminuição da capacidade do indivíduo em resistir ou adaptar-se ao estressor, podendo surgir leves problemas de saúde, que não o incapacitam.

As respostas do estresse englobam mecanismos hormonais que se iniciam com o estímulo da neuroipófise no cérebro e de inúmeros eventos que envolvem glândulas da suprarrenal, tendo ação no estômago, coração e sistema linfático e atingindo, principalmente, o sistema imunológico, que tende a ficar comprometido, diminuindo os níveis de endorfina e serotonina, responsáveis pela elevação da autoestima do ser humano (PRETO VA, et al., 2018).

\section{Conceito de estresse}

As doenças psíquicas têm crescido mundialmente em todas as classes sociais, sexo e idade. Esse assunto vem despertando interesse em pesquisadores e o estresse é uma das doenças psíquicas desse grupo de maior prevalência (CESTARI VRF, et al., 2017).

O tema estresse é discutido em todos os níveis da vida humana. Ele é descrito como um processo negativo, causando agravos na função multiorgânica do indivíduo doente tais como desordens físicas, mentais, de personalidade e imunológicas, que irão prejudicar o exercício da profissão, a saúde, o bem-estar e as relações interpessoais.

De acordo com estudos avaliados, cada sujeito desenvolve o estresse segunda suas vivências e condições predeterminadas. Sendo assim, o estresse pode ser avaliado de diferentes formas em cada indivíduo de acordo com sua adaptação ao meio em que se relaciona (PEREIRA CA, et al., 2010; HIRSCH CD, et al., 2018).

Nesse sentido Oliveira LA, et al. (2014), relatam que é importante conhecer e ajustar-se ao ritmo acelerado da vida, aos desgastes diários, as muitas exigências, manter uma alimentação efetiva e saudável a ausência de tempo para as atividades de lazer e repouso, pois essas condições favorecem o aparecimento do estresse. 
O estresse, no enfoque psicológico é conceituado como um somatório de ações que os indivíduos se expõem desenvolvendo sintomas transitórios ou permanentes (NODARI NL, et al., 2014). Vários agravos a saúde estão relacionados ao estresse e o aparecimento dos sintomas não é a principal manifestação, mas uma resposta multiorgânica contribuindo para o desequilíbrio biológico, psíquico e social (BENAVENTE, SBT e COSTA ALS, 2011).

\section{Sinais e sintomas do estresse}

Quando o indivíduo se expõe a uma situação de estresse permanente, ou por tempo prolongado, ela pode acarretar inúmeras consequências físicas e psicológicas tais como: o aumento da pressão arterial e maior suscetibilidade ao Acidente Vascular Cerebral (AVC); quadros de infecção devido à diminuição da resposta imunológica; distúrbios gastrointestinais como a diarreia e a constipação; desordens alimentares; ganho ou perda excessiva de peso; resistência à insulina associada ao diabetes tipo 2 e à exacerbação do diabetes (PRETO VA, et al., 2018).

Estresse é uma experiência de tensão, irritação onde o organismo reage a componentes físicos ou psicológicos quando há uma situação que provoque medo, excitação ou confusão (TORQUATO JÁ, et al., 2010). Para Murakami K, et al. (2019) os sintomas do estresse são diversos, dependendo da fase em que se encontra.

Lipp M, (2015) descrevem que os sintomas físicos podem se manifestar por meio de sinais e alterações gastrointestinais; problemas cardiovasculares, tais como taquicardia, hipertensão arterial, formigamento em membros superiores e inferiores; problemas dermatológicos, mal-estar geral; entre outros.

As manifestações psicológicas se caracterizam principalmente por alterações de humor, depressão, angústia, ansiedade, irritabilidade, apatia, tiques, problemas de relacionamento, distúrbios do sono e dificuldades de concentração (apud Santana LL, et al., 2018).

Os sinais e sintomas mais comuns observados no estresse podemos destacar a cefaleia do tipo tensional; insônia; desejo sexual diminuído e impotência temporária nos homens; exacerbação da tensão pré-menstrual nas mulheres, além da diminuição da concentração; inibição do aprendizado; redução da memória, bem como a exacerbação de lesões de pele e depressão, aumento da sudorese, tensão muscular, taquicardia, hipertensão arterial, aperto da mandíbula, ranger de dentes, hiperatividade, náuseas, mãos e pés frios (DORMANN C, ZAPF D, 2002; SAMPAIO RFV, et al., 2012 apud PRETO VA, et al., 2018).

Os acadêmicos de enfermagem estão cercados diariamente por conflitos, devido estar em contato com a dor e o sofrimento humano, podendo acarretar sentimento de insegurança, que consequentemente afetam a maneira em que percebem sua qualidade de vida (BARBOSA T, et al., 2016).

O estudo do estresse em acadêmico vem sendo debatido em várias áreas do conhecimento pois, ao mesmo tempo que esses universitários adquirem conhecimento e informação para atuarem em suas profissões estes são expostos a situações de frustação, temores e angústias e a convivência com esse ambiente por vezes, pode desencadear distúrbios patológicos (GERVÁSIO SM, et al., 2012 apud CESTARI VRF, et al., 2017).

Benavente SBT e Costa ALS (2011), contribuem com a identificação entre os estudantes de enfermagem as manifestações físicas do estresse como transpiração, tremores e debilidade física e as manifestações psicológicas de pânico, angústia, solidão e abandono, tristeza, desmotivação, frustração, impotência e revolta; foi verificada a prevalência de angústia perante todas as manifestações mencionadas pelos estudantes.

\section{$O$ estresse em acadêmicos}

Com a organização das Diretrizes Curriculares Nacionais para o Ensino de Graduação em Enfermagem, vigente desde 2001, amparando todos os princípios, os fundamentos, as condições e os procedimentos para a formação de enfermeiros, onde além de determinar os conteúdos curriculares, teóricos e práticos, insere a obrigatoriedade do estágio supervisionado em ambientes hospitalares, ambulatórios, serviços da rede básica de saúde e atuação nas comunidades nos dois últimos semestres do Curso de Graduação em Enfermagem (LIMA TC, et al., 2014). 
Para Hirsch CD, et al. (2018) o estresse, pode acontecer em momentos diferentes da vida de um indivíduo, mas, a mudança de rotina e as exigências de um curso superior podem desencadear o estresse nos estudantes. A literatura aponta que o estresse pode gerar consequências negativas aos estudantes universitários, como o surgimento de transtornos de ansiedade e de humor. Elevados níveis de estresse estão relacionados ao abuso de drogas, dificuldade de relacionamentos interpessoais, depressão e suicídio na população de universitários (MURAKAMI K, et al., 2019).

Os alunos durante a sua trajetória universitária vivenciam ambientes e situações novas e diferentes da realidade da vida, necessitando de ajustes para essas novas condições acadêmicas, esses fatores podem servir como desencadeadores de estresse ou de ansiedade (SANTOS RJLL, et al., 2019).

No entanto, muitas vezes, essa sucessão de novas situações vivenciadas pelos acadêmicos pode exceder a capacidade de adaptação e serem avaliadas como estressoras, dificultando o processo de aprendizagem $e$ influenciar no bem-estar do estudante (BUBLITZ S, et al., 2012).

Para o desenvolvimento de habilidades, segurança e aperfeiçoamento de técnicas os acadêmicos realizam estágios supervisionados que é visto como um importante indicador para a formação dos profissionais de enfermagem no exercício da profissão. Essa atividade prática serve para fortalecer o aprendizado teórico, formando profissionais mais capacitados para o enfrentamento do competitivo mercado de trabalho (DIAS EP, et al., 2014).

Oliveira LA, et al. (2014) contribuem afirmando que o acadêmico de enfermagem, ao iniciar suas atividades passam por várias situações adversas tais como, a formação de um novo ciclo de amigos, adaptação a novos horários, problemas financeiros, preocupações com seu futuro e mercado de trabalho, afastamento de seu ambiente familiar, as cobranças, o encontro com a dor, o sofrimento, a doença e a morte de pessoas queridas podendo gerar conflitos internos e sentimentos dúbios.

Estes fatores são vistos como estressores, que irão estartar o estresse complicando sua adaptação a essas novas situações. Em um estudo comparando os acadêmicos universitário iniciantes com os concluintes, observou-se a diferença entre as duas populações. Os iniciantes vivenciam várias situações de estresse nesse ambiente novo até a sua adaptação (BUBLITZ S, et al., 2016 apud MUSSI FC, et al., 2019).

Alguns atributos que favorecem ao estresse são destacados em estudos realizados em universitários de enfermagem, tais como características socioeconômicas como sexo, faixa etária, estado civil e renda familiar, gastos com moradia, a formação, além de outras despesas pessoais e a renda mensal (PRETO VA, et al., 2018 apud RIBEIRO FMSS, et al., 2019). Mussi FC, et al. (2019) relatam que várias situações podem prejudicar a saúde e a qualidade de vida dos acadêmicos, como, o gerenciamento da vida pessoal e social, as exigências acadêmicas, a preparação para o início da carreira profissional e as responsabilidades financeiras.

Os autores Pacheco S, (2008), Bowden J, (2008) também descrevem essas fontes de estresse nos estudantes de enfermagem, e citam que as atividades práticas e os estágios, devido a carga horária longa, avaliações constantes, elaboração de relatórios e redação de forma correta são exemplos de fontes de estresse para esses estudantes (apud Benavente SBT e Costa ALS, (2011).

Em especial no ambiente acadêmico, a resolução de problemas é essencial. Além disso, os estudantes passam por momentos de mudança, desenvolvimento, frustração, crescimento, temores e angústias. Neste contexto o ambiente que deveria contribuir para somar conhecimentos passa a ser um motivador de distúrbios patológicos (SANTANA LL, et al., 2018).

Nos estudos analisados há a inferência que no processo ensino e aprendizagem, o aluno experimenta situações que podem ser ameaçadoras ou desafiantes e essa adaptação é uma constante preocupação dos estudantes ao longo de todo o curso de graduação. (MORA VRH, HERRERA RL, 2004; TULLY A, 2004 apud BENAVENTE SBT e COSTA ALS, 2011).

Cabe ressaltar também que, ao lidar com os limites humanos, alguns dos acadêmicos apresentam dificuldades e desenvolvem sentimentos de incapacidade frente às atividades exigidas durante a formação profissional, o que favorece a diminuição do seu rendimento acadêmico e interfere na qualidade da assistência 
de enfermagem prestada durante os estágios. Inicia-se, assim, uma situação na qual a instabilidade emocional pode influenciar na avaliação das demandas curriculares e levar estes graduandos a considerar 0 processo de formação estressante (BUBLITZ S, et al., 2012).

Para melhorar os níveis de estresse podem ser ofertadas no espaço universitário, a prática de atividade física, sessões de relaxamento ou a auriculoterapia, já evidenciada como eficaz para a diminuição do nível de estresse, bem como atendimento psicopedagógico (RIBEIRO FMSS, et al., 2019).

\section{CONSIDERAÇÕES FINAIS}

A partir desse estudo verificamos que o estresse está presente em todos os períodos de formação acadêmica, com tendência a se intensificar com o início das atividades práticas de ensino clínico. De acordo com a literatura, quanto mais avançado o período em que o aluno se encontra, maior seu nível de estresse, uma vez que a preocupação com o conhecimento adquirido e seu impacto em sua futura vida profissional aumentam ao longo desse tempo. Portanto, o desenvolvimento de métodos de orientação e treinamento para prevenir o estresse de acadêmicos no campo de estágio deve ser uma meta centrada nos cursos de Graduação em Enfermagem.

\section{REFERÊNCIAS}

1. BARBOSA T, et al. Percepção do estresse e qualidade de vida dos acadêmicos de enfermagem no Brasil. Revista Repositório Científico de Acesso Aberto de Portugal, 2016, v 4, I2; 94-102.

2. BENAVENTE SBT, COSTA ALS. Respostas fisiológicas e emocionais ao estresse em estudantes de enfermagem: Revisão Integrativa da literatura científica. Revista Acta Paulista, 2011, 24(4): 571-6.

3. BUBLITZ S, et al. Estressores entre acadêmicos de enfermagem de uma Universidade Pública. Revista de Enfermagem da UERJ, 2012; 20(2): 739-45.

4. BUBLITZS, et al. Estresse em estudantes de enfermagem: uma revisão integrativa. Revista de enfermagem da UFSM, 2012; 2(3): 530-538.

5. CESTARI VRF, et al. Estresse em estudantes de Enfermagem: Estudo sobre a vulnerabilidade sociodemográfica e acadêmica. Revista Acta Paulista de Enfermagem, 2017: 30(2): 190-6.

6. DIAS EP, et al. Expectativas de alunos de enfermagem frente ao primeiro estágio em Instituições de Saúde. Revista Psicopedagogia, 2014: 33(94): 44-55.

7. HIRSCH CD, et al. Fatores percebidos pelos acadêmicos de enfermagem como desencadeadores do estresse no ambiente formativo. Revista Texto e Contexto Enfermagem, 2018; 27(1): e0370014.

8. LIMA TC, et al. O estágio curricular supervisionado: Análise da Experiência discente. Revista Brasileira de Enfermagem (REBEN), 2014; 67(1): 133-40.

9. MURAKAMI K, et al. Estresse Psicológico em estudantes do curso de graduação da área da saúde: Subsídios para promoção de Saúde Mental. Revista Med. (São Paulo), 2019; mar-abr: 98(2) 108-13.

10. MUSSI FC, et al. Comparação do estresse em universitários de enfermagem ingressantes e concluintes do curso. Revista da Escola de Enfermagem da USP, 2019; 53 e 03431.

11. NODARI NL, et al. Estresse, conceitos, manifestações e avaliação em saúde: Revisão da Literatura. Revista Saúde e Desenvolvimento Humano, 2014. maio 30: 2(1): 61-74.

12. OLIVEIRA LA, et al. Estresse nos acadêmicos de enfermagem de uma universidade pública. Revista Arquivo de Ciências da Saúde, 2014; 21(2) 118-23.

13. PEREIRA CA, et al. O estresse e seus fatores determinantes na concepção dos graduandos de enfermagem. Revista Mineira de Enfermagem, 2010,14(2): 204-209, abril-junho.

14. PRETO VA, et al. Percepção de estresse nos acadêmicos de enfermagem. Revista de Enfermagem da UFPE on line. Recife, 2018, 12(3): 708-15, mar.

15. RIBEIRO FMSS, et al. Estresse em Universitárias(os) de enfermagem e a necessidade de enfrentamento. Revista Científica Sena Aires, 2019 out-dez, 8(1): 1-3.

16. SANTANA LL, et al. Estresse no cotidiano de graduação de enfermagem de um Instituto Federal de Ensino. Revista de Enfermagem do Centro-Oeste Mineiro, 2018: 8, v8. E2738.

17. SANTOS RJLL, et al. Estresse em acadêmicos de enfermagem: Importância de identificar o agente estressor. Brazilian Journal of health Review. Curitiba, 2019, v2, no 2, p6, 1086-1094, mar/abr.

18. TORQUATO JA, et al. Avaliação do estresse em estudantes universitários. Revista Científica Internacional, 2010, Ano $3, n^{\circ} 14$, julho-agosto. 